\title{
Systematic observations of anomalous refraction at millimeter wavelengths
}

\author{
L. Olmi ${ }^{\star}$ \\ LMT/GTM Project, Dept. of Astronomy, 815J Lederle GRT Tower B, University of Massachusetts, \\ 710 N. Pleasant st., Amherst, MA 01003, USA
}

Received 6 March 2001 / Accepted 27 April 2001

\begin{abstract}
It is well known that the water vapor in the troposphere plays a fundamental role in radio propagation. The refractivity of water vapor is about 20 times greater in the radio range than in near-infrared or optical regimes. As a consequence, phase fluctuations at frequencies higher than about $1 \mathrm{GHz}$ are predominantly caused by fluctuations in the distribution of water vapor, and thus radio seeing at these frequencies is predominantly caused by tropospheric turbulence. Radio seeing shows up on filled-aperture telescopes as an anomalous refraction (AR), i.e. an apparent displacement of a radio source from its nominal position, corrected for large-scale refractive effects. The magnitude of this effect, as a fraction of the beam width, is bigger on larger telescopes and thus its impact on the pointing is likely to become critically important in the next generation of electrically large filledaperture radio telescopes $\left(D / \lambda>10^{4}\right)$ and in particular on the Large Millimeter Telescope. AR effects are expected to reduce the total effective observing time at the highest frequencies and will affect on-the-fly mapping. Here we present the results of systematic AR measurements carried out with the 13.7-m telescope of the Five College Radio Astronomy Observatory. The measured AR pointing errors range from $1^{\prime \prime}-3^{\prime \prime}$ (winter) to about $20^{\prime \prime}$ (summer) and most of the events last less than about $4 \mathrm{~s}$. We analysed the structure function, power spectrum and Allan variance of the data and we have carried out a statistical analysis to identify correlations of the statistical functions with selected observing parameters such as precipitable water vapor, time of day, season and elevation angle. Our results suggest that uncompensated AR may be the most important dynamic environmental source of pointing errors on the new large radio telescopes (ALMA, GBT, LMT, SRT) and may guide the design of active AR-compensation devices and help allocating suitable observing time through dynamic scheduling.
\end{abstract}

Key words. atmospheric effects - methods: observational - telescopes

\section{Introduction}

Radio-seeing effects on centimeter- and millimeterwavelength interferometers are a consequence of the inhomogeneously distributed atmospheric water vapor which can cause spatial and temporal variations in the optical path length of radio waves. Several studies of the problem of phase fluctuations with both centimeter (e.g., Armstrong \& Sramek 1982) and millimeter (e.g., Bieging et al. 1984; Olmi \& Downes 1992; Wright 1996) interferometers have led to the development of a number of radiometric devices to compensate for these fluctuations and restore the uncorrupted phase off-line (e.g., Bremer 1995; Marvel \& Woody 1998).

On the other hand, radio seeing on filled-aperture telescopes shows up as an anomalous refraction (AR), i.e., an apparent displacement of a radio source from its true position, caused by the phase difference introduced between the opposite extremities of the receiving aperture because

\footnotetext{
* e-mail: olmi@lmtgtm.org
}

the propagation paths traverse air masses of varying humidity. AR pointing effects caused by turbulence in the "wet" atmosphere are similar to the "quivering" of stars observed with visual-wavelength telescopes, which are also known as angle of arrival fluctuations in the field of clear- or dry-air propagation effects (see, e.g., Fante 1975; Lawrence \& Strohbehn 1970). The magnitude of this effect, as a fraction of the beam width, is bigger on larger telescopes and thus its impact on the pointing is likely to become critically important in the next generation of electrically large filled-aperture radio telescopes $(D / \lambda>$ $10^{4}$ ), and especially in the case of the Large Millimeter Telescope (or "Gran Telescopio Milimetrico", in Spanish, LMT/GTM; see Olmi 1998; Kaercher \& Baars 2000) with a $F W H M \simeq 5^{\prime \prime}$ at $\lambda=1 \mathrm{~mm}$ and a required pointing accuracy at this wavelength $<1^{\prime \prime}$.

The first extensive measurements of AR were carried out by Altenhoff et al. (1987), Downes \& Altenhoff (1990), and also Church \& Hills (1990) who found that AR events are characterized by angular displacements of the sources 
from their true positions by a few arc seconds, in both azimuth and elevation, for a few seconds of time, but occasionally showing much larger events that could last for tens of seconds. This is similar to what is observed in near-infrared astronomy, where, for small telescope diameter to Fried parameter ratio, $D / r_{\circ}(\lambda) \lesssim 6$ (the Fried parameter represents the seeing cell size), the short-exposure point spread function (PSF) randomly moves in the focal plane (e.g., Close \& McCarthy 1994). On the new large radio telescopes that are either under construction $\left(\mathrm{GBT}^{1}, \mathrm{LMT}\right)$ or beeing designed (ALMA, $\mathrm{SRT}^{2}$ ), for which $D / r_{\circ}(\lambda) \gtrsim 1$ at the highest frequencies, phase gradients across the antenna aperture (i.e., tilt) will dominate, but there will be also higher order aberrations that can effectively broaden the primary beam (Olmi 2000a). In more recent years these projects have also prompted serious investigations of techniques to compensate AR effects (see Holdaway 1997; Butler 1997; Holdaway \& Woody 1998; Olmi 2000a, 2000b).

There were therefore several reasons to carry out an extensive, systematic study of the AR effects using a singledish antenna: (i) AR is the most critical dynamic environmental source of pointing errors on large millimeter and submillimeter telescopes; (ii) the measurements of phase fluctuations with millimeter interferometers and "seeing monitors" is sensitive to the relative orientation of the baseline and the wind direction (Lay 1997), and they are often carried out over large spatial scales compared to the diameter of single-dish antennas; (iii) it is important to determine the potential effects of AR during On-The-Fly (OTF) mapping; (iv) the next generation of mm-wave telescopes represent big time, effort, and money investments and thus must meet their design goals and yield a high observing efficiency; $(v)$ a better knowledge of AR would also improve the design of active AR-compensation devices and help allocating suitable observing time through dynamic scheduling.

The main goal of this work is to present the results of systematic AR observations carried out with the 13.7-m telescope of the Five College Radio Astronomy Observatory $^{3}$ (FCRAO) located in New Salem (USA) at an elevation of $314 \mathrm{~m}$ above sea level. They show that AR is clearly detectable with the FCRAO $60^{\prime \prime}$ beam-width at $86 \mathrm{GHz}$ even when the precipitable water vapor $(P W V)$ is a few $\mathrm{mm}$ only. Measured values range from as "little" as $1^{\prime \prime}-2^{\prime \prime}$ (winter) to as much as $20^{\prime \prime}$ (summer). The main purposes of these observations were: (i) detect AR effects and characterize their magnitude (and time-scales) as a function of time of the day, season, and elevation; (ii) detect and measure systematic changes in AR statistical properties (slopes, turn-overs, etc.). Some results

\footnotetext{
1 "Green Bank Telescope".

2 "Atacama Large Millimeter Array", "Sardinia Radio Telescope".

${ }^{3}$ The Five College Radio Astronomy Observatory is operated with support from the National Science Foundation and with permission of the Metropolitan District Commission.
}

from an incomplete data sample can be found in Olmi (2000b, 2001) where we also discuss the basic technical problems of a tip-tilt compensation device at millimeter wavelengths for the LMT as well as other related issues. The outline of the paper is as follows: in Sect. 2 we describe the measurement technique; in Sect. 3 we analyze and discuss the AR data using several statistical functions; finally, we draw our conclusions in Sect. 4.

\section{Observations}

The AR observations have been carried out using the FCRAO radome-enclosed 13.7-m telescope located in western Massachusetts. The telescope site is characterized by flat terrain surrounded by woods, with $P W V$ values (calculated using the measured ground-level dew point temperature) ranging from $<1 \mathrm{~mm}$ in winter to more than $10 \mathrm{~mm}$ in summer time. The occurrence of AR was recorded by tracking a strong $\mathrm{SiO}$ maser $(\nu=86 \mathrm{GHz})$ pointlike source at the azimuth half-power points of the response pattern. The source intensity was then compared with the ON-source intensity to determine the apparent angular shift, assuming a given main beam pattern that is well represented by a Gaussian profile (Ladd \& Heyer 1996). There is a tendency for the beam to be broader at lower elevations, because of (mainly) gravitational effects, but at $86 \mathrm{GHz}$ the maximum $F W H M$ variation is about $\pm 5 \%$ for elevations $\gtrsim 30^{\circ}$ and is $\pm 8 \%$ for elevations between $20^{\circ}$ and $30^{\circ}$ (Ladd \& Heyer 1996). Because the pointing errors are obtained through a relative measurement they are not affected by gain variations as a function of elevation angle.

The pointing and focus of the telescope were checked at the beginning of a new observing session and about every 30 min thereafter. The typical absolute pointing accuracy was about $6^{\prime \prime}$, although the critical parameter of interest to AR measurements is the tracking accuracy (see below). Likewise the ON-source intensity was checked before and after an AR time series. Using this technique one measures the modified angular distance, $\theta$, of the target source from the beam center, i.e. $\Delta \theta=\theta-\theta_{\circ}$, where $\theta_{\circ}$ is the beam $F W H M$ (see Fig. 1 of Olmi 2000b). $\Delta \theta$ is the quantity of interest to determine the antenna pointing error, and the data used in this work are time series of the observable $\Delta \theta(t)=\theta(t)-\theta_{\circ}$. Further information about the observing technique can be found in Olmi (2000b).

Two sets of data were obtained: a large sample where the source intensity was sampled every $\tau_{\mathrm{s}}=3 \mathrm{~s}$, for as long as $5.5 \mathrm{~min}$, and a smaller set of data with $\tau_{\mathrm{s}}=1 \mathrm{~s}$ and with durations of up to $10 \mathrm{~min}$. The data sampled with $\tau_{\mathrm{s}}=3 \mathrm{~s}$ were obtained under cloud-free conditions in the period of February 1999 to June 2000 and we will refer to them as sample I. The data with $\tau_{\mathrm{s}}=1 \mathrm{~s}$ were obtained during similar and, very often, during the same weather conditions as sample I during spring 2000, and we will refer to them as sample II. The values of the outside temperature and $P W V$ were recorded during the observations. However, no data on wind speed and direction 

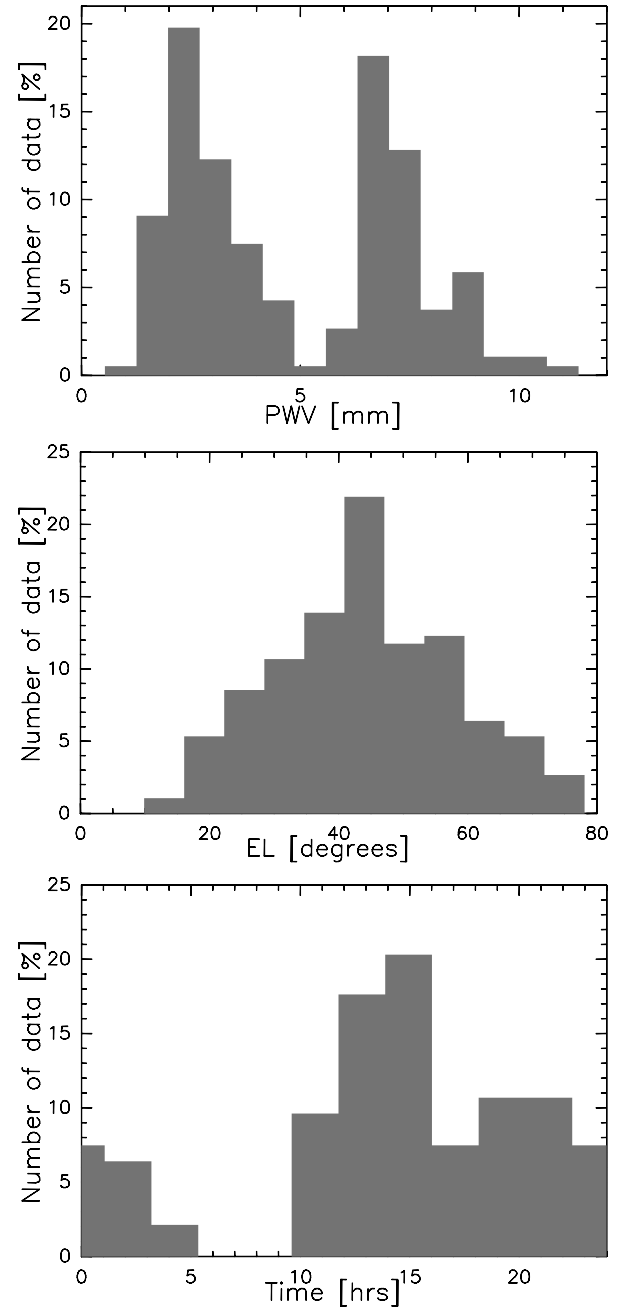

Fig. 1. Histograms showing the distribution of the data as a function of PWV (top), elevation angle (middle) and local time (bottom), for combined data sample I and II.

were available. The data are not uniformly distributed in the observing parameters' space. In particular, most of the data have been taken during conditions of either low or high $P W V$, as shown in Fig. 1, due to the availabilty of observing time during the regular observing season (when typically $P W V \lesssim 5 \mathrm{~mm}$ ) and during the month of June before the receivers are shut down.

The range of spatial frequencies analyzed was $\simeq 0.003 \mathrm{~Hz}$ to a Nyquist frequency of $f_{\mathrm{N}}=0.167 \mathrm{~Hz}$ for sample I and $\simeq 0.002 \mathrm{~Hz}$ to $f_{\mathrm{N}}=0.5 \mathrm{~Hz}$ for sample II. Consequently, we were unable to observe AR events with time scales shorter than $\tau_{\mathrm{s}}=1 \mathrm{~s}$. Our measurements carried out during very dry conditions in winter $(P W V<1 \mathrm{~mm})$ indicate that the telescope tracking error is $<1^{\prime \prime}$, which we then consider as the sensitivity limit of our AR observations. No correlation was found between the AR pointing error and various recorded telescope parameters, such as subreflector's motors readings and the electronic levels of the AZ track.

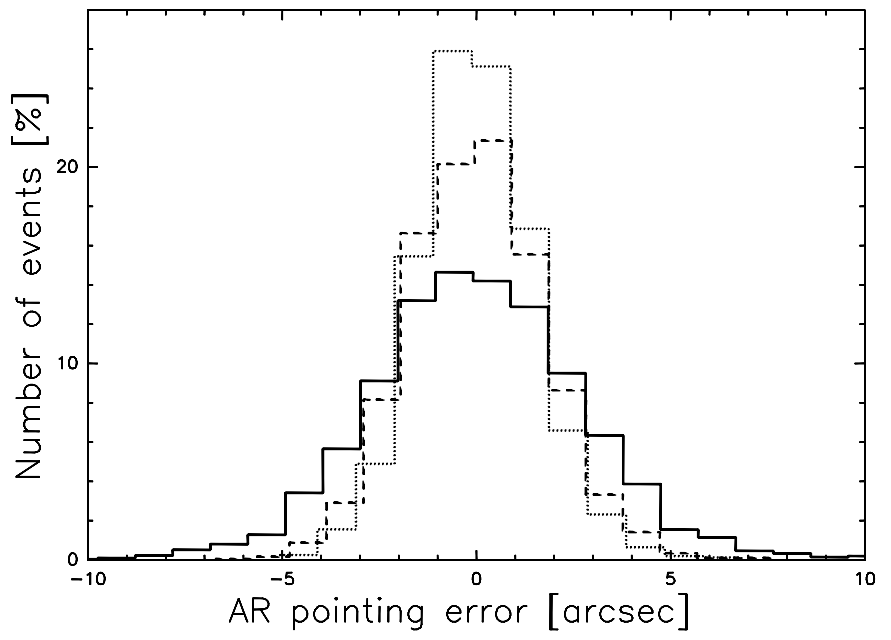

Fig. 2. Histograms of AR-induced angular displacements $(\Delta \theta)$ observed on different days: $13-\mathrm{APR}-2000$ with $P W V=2.7 \mathrm{~mm}$ (dotted line) corresponding to an optical depth at $225 \mathrm{GHz}$ of $\tau_{225}=0.27 ; 14$-APR-2000 with $P W V=4.0 \mathrm{~mm}$ or $\tau_{225}=0.33$ (dashed line); and 26-MAY-2000 with $P W V \simeq 7.2 \mathrm{~mm}$ or $\tau_{225}=0.59$ (solid line). The bins have a width $\simeq 1^{\prime \prime}$.

\section{Results and analysis}

\subsection{Probability density distribution}

Figure 2 shows the distribution of angular displacements observed on three days with different $P W V$ of sample II. The data were taken during a short period of time (between 13:00 and 16:00 local time) in stable weather and thus during conditions of very similar $P W V$ and temperature. Because the three histograms in Fig. 2 are not the average of many days of data, with very different conditions, they represent a good approximation of the AR probability density function (PDF) for a given $P W V$. Moreover, the air-mass range was approximately the same for each of the three days considered (1.1 to 1.6) and therefore the different widths of the histograms cannot be explained as an elevation effect (see Sect. 3.5 for a discussion about elevation effects).

The widths of the PDFs in Fig. 2 corresponding to about $75 \%$ of the total area underneath the histograms are $\simeq 6^{\prime \prime}$ and $\simeq 4^{\prime \prime}$ for the 26 -MAY-2000 and the April 2000 data, respectively. Therefore, during typical dry weather $\left(P W V<4 \mathrm{~mm}\right.$, or $\tau_{225}<0.33$ at the FCRAO site) the magnitude of AR can be of up to several arcseconds, although we cannot exclude that it might be even larger on time-scales shorter than $\tau_{\mathrm{s}}$. In summer time at FCRAO, or during conditions of high $P W V$, the AR pointing error can be a considerable fraction of the FCRAO beam-width. All other observing conditions being approximately the same, the $P W V$ seems to be a good tracer of AR activity (but see the important discussion in Sect. 3.5) and may thus allow an extrapolation of these results to other sites. We note, however, that we obtained these values on a flat terrain and there is certainly more turbulence on a mountain top. Therefore, under similar $P W V$ conditions we might expect more variations in the AR-induced pointing 


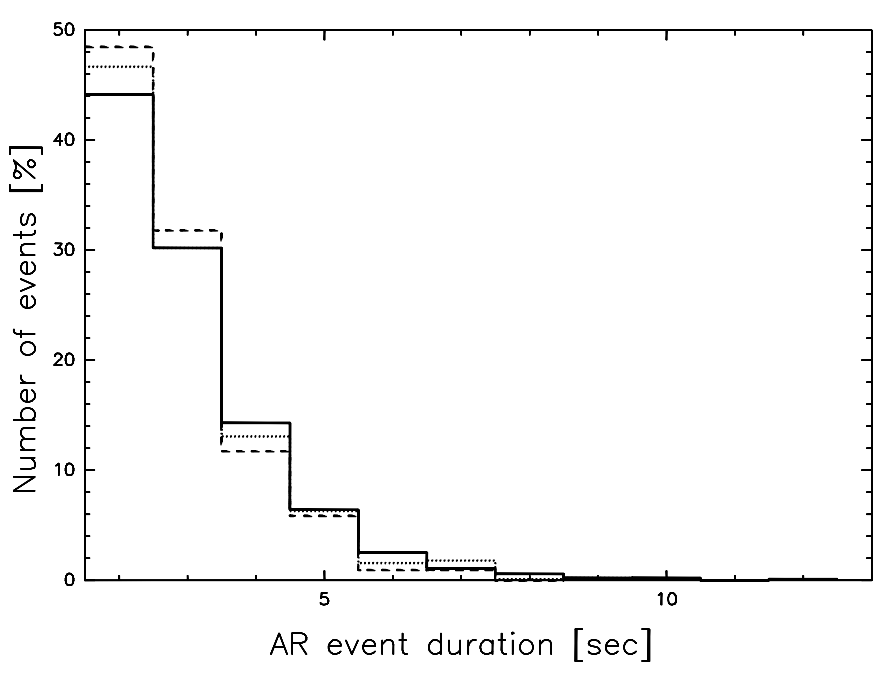

Fig. 3. Histograms of the duration of AR events for the same days shown in Fig. 2 (line styles are the same). The bins have a width $\simeq 1 \mathrm{~s}$.

errors measured on the LMT/GTM site, due to its more complex topography, than on a large open site such as the FCRAO. Furthermore, because the largest AR-induced pointing errors will occur at the shortest wavelengths, and because it is likely that the LMT/GTM will operate in this high-frequency regime only during conditions of low ( $\$ 5-10 \mathrm{~m} / \mathrm{s}$ ) wind-speed, we should not expect that the reduced time-scale of AR events resulting during conditions of high wind-speed will contribute to average the AR effects down. It is clear, however, that extrapolating these results to sites with different characteristics and at higher frequencies is difficult as well as uncertain and specific on-site measurements should be obtained.

\subsection{Duration of $A R$ events}

We define the duration of an "AR event" as the time interval between two measurements, preceeding and following a peak (either positive or negative) in the AR time series, and having a value of $\Delta \theta$ smaller than $50 \%$ of the peak value. In Fig. 3 we show the distribution of the durations of the apparent displacements of the source as observed on the same days as in Fig. 2. Two interesting features can be seen: first, the three histograms are remarkably similar and do not show any specific feature associated with different $P W V$ s. Second, most $(\gtrsim 75 \%)$ of the events last less than about 3-4 s. To first order, the typical duration of an AR event is consistent with the time it takes a moist element to cross the dish and it is expected to be longer on larger antennas (see Holdaway 1997). Moreover, the distribution of the event durations has a tail which may stretch to times $\sim 10-20 \mathrm{~s}$, although these events are much rarer. The AR events durations obtained using data from sample I have similar distributions but they fail to show that most of the events are of very short duration (see Olmi 2000b). Therefore, observations that are short compared to the typical duration of the AR events, as is the case in OTF mapping, will be seriously affected by the AR pointing errors. Multiple sweeps across the source may somewhat reduce the average pointing error but will incur in a flux density loss and primary beam broadening anyway.

Are the longest AR events also the ones with larger magnitudes? To answer this question we generated the three-dimensional plots shown in Fig. 4 where the distribution of the data points is plotted as a function of the AR-induced pointing error and the corresponding event duration. The double-peak structure is due to the equal probability of having, for each duration time, either a positive (the angular distance of the target source from the center of the beam increases as an effect of AR) or negative (the target source approaches the beam center) pointing error. Clearly, the PDFs of the AR pointing errors are very similar at any given event duration, except of course for the total number of occurrences that decreases for longer durations as already discussed above. Therefore, our data show that while AR events of short duration are more likely to occur, the distribution of their magnitudes remains approximately constant. Multiple events that would show up as long duration ones are possible but they would be indistinguishable from individual events and we have not attempted any sophisticated procedure to select them.

\subsection{AR structure function}

\subsubsection{Observed data}

The temporal structure function, $\mathcal{D}_{\theta}(\tau)$ where $\tau$ is the time lag, for the observable AR-induced pointing error $\Delta \theta(t)$ can be defined as (Olmi 2000b):

$\mathcal{D}_{\theta}(\tau) \equiv\left\langle[\Delta \theta(t+\tau)-\Delta \theta(t)]^{2}\right\rangle=c_{\theta}^{2} \tau^{\alpha}$

where the second equality indicates that in most cases the structure function, or parts of it, can be fit using a power law. In this case the slope of the structure function in a $\log$-log plot, $\alpha$, is a measure of how rapidly the intensity of the fluctuations of $\Delta \theta$ increases with increasing $\tau$; the parameter $c_{\theta}^{2}$ is called the structure function coefficient and is a direct measure of the strength of the AR effects. We show two examples of measured structure functions from sample II in Fig. 5; in particular, the top panel shows an example of saturation of the AR structure function, i.e. a flattening of the slope at higher lag times and thus a break in the slope, whereas the bottom panel shows an example of structure function that can be fit with a single power law.

The distribution of $\alpha$ and of the intercept, $\log c_{\theta}{ }^{2}$, are shown in Fig. 6 for the entire set of data, i.e. samples I and II. Most of the slopes of $\mathcal{D}_{\theta}(\tau)$ are $\lesssim 0.4$ and the weighted average is $\langle\alpha\rangle=0.2$. However, $\log c_{\theta}^{2}$ can vary over two orders of magnitude from about -4 to about -2 . 


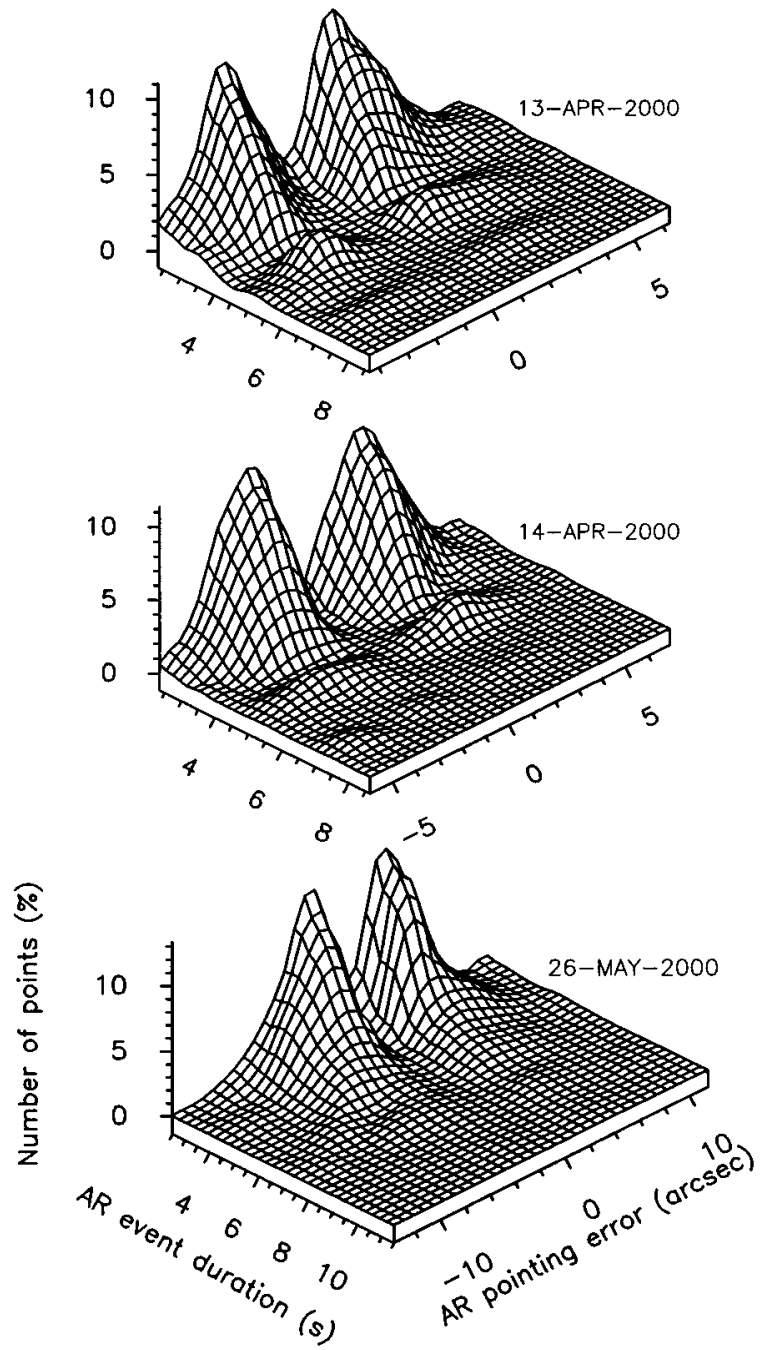

Fig. 4. Distribution of the data points as a function of the event duration and pointing error, for the same days as in Fig. 2.

\subsubsection{Comparison with phase structure function}

The AR structure function and the phase structure function measured with interformeters are different, and this may also explain why in many cases the AR structure function can saturate as shown in Fig. 5. The spatial phase structure function at a given time $t$ is defined as:

$\mathcal{S}_{\phi}(b) \equiv\left\langle[\phi(r+b, t)-\phi(r, t)]^{2}\right\rangle$

where $\phi$ is the wavefront phase measured at two positions separated by the distance $b$. If we replace the baseline $b$ with the distance separating two points on the antenna diameter $D$ and assume that the wavefront across the antenna is tilted with respect to the optical axis but has no higher-order aberrations otherwise, then the phase difference, $\Delta \phi(t)$, across the antenna aperture can be approximated as:

$\Delta \phi(t) \equiv \phi(r+D, t)-\phi(r, t) \simeq \frac{2 \pi}{\lambda} D \Delta \theta(t)$

where $\Delta \theta(t)$ is the instantaneous tilt of the wavefront, equivalent to the pointing error. The procedure for finding

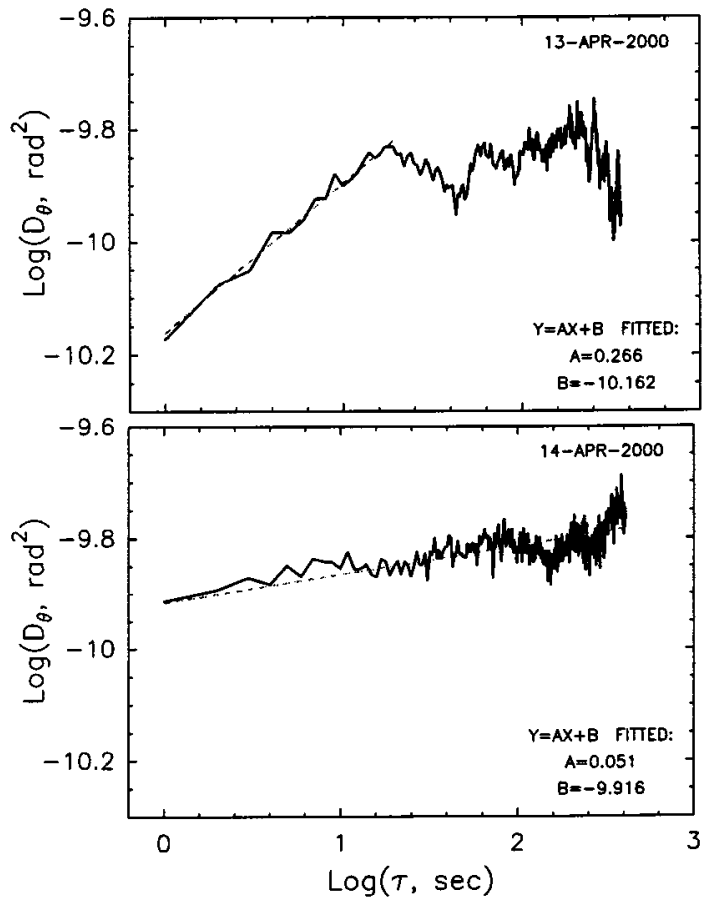

Fig. 5. Top: example of structure function with a break in the slope and saturation at longer lag times. Bottom: example of structure function with no saturation.

the relationship between $\mathcal{S}_{\phi}(D)$ and $\mathcal{D}_{\theta}(\tau)$ involves the use of Taylor's hypothesis of frozen turbulence so that (Lawrence \& Strohbehn 1970):

$\phi(r, t+\tau)=\phi(r-v \tau, t)$

where $v$ is the average wind velocity component perpendicular to the line-of-sight of the imaging system. We can now relate $\mathcal{S}_{\phi}(D)$ and the temporal phase structure function, $\mathcal{D}_{\phi}(\tau)$ :

$\mathcal{D}_{\phi}(\tau) \equiv\left\langle[\phi(r, t+\tau)-\phi(r, t)]^{2}\right\rangle=\mathcal{S}_{\phi}(v \tau)$.

Using Eqs. (1) and (3) one can then write the AR structure function as:

$$
\begin{aligned}
\mathcal{D}_{\theta}(\tau)= & \frac{1}{k D}<([\phi(r+D, t+\tau)-\phi(r, t+\tau)] \\
& -[\phi(r+D, t)-\phi(r, t)])^{2}>
\end{aligned}
$$

where $k=2 \pi / \lambda$. We can then use Eqs. (2) to (4) in Eq. (6) to finally obtain the relationship between phase and AR structure functions:

$$
\begin{aligned}
\mathcal{D}_{\theta}(\tau)= & \frac{2}{k D}\left\{\mathcal{S}_{\phi}(D)+\mathcal{S}_{\phi}(v \tau)\right. \\
& \left.-\frac{1}{2}\left[\mathcal{S}_{\phi}(|D-v \tau|)+\mathcal{S}_{\phi}(D+v \tau)\right]\right\}
\end{aligned}
$$

where we have dropped the time dependence and we have also assumed that the turbulence is isotropic. Depending on the value of $v \tau$ two different regimes can be defined in Eq. (7):

$\mathcal{D}_{\theta}(\tau) \simeq \begin{cases}\frac{2}{k D} \mathcal{S}_{\phi}(D) & \text { if } v \tau>>D \\ \frac{2}{k D} \mathcal{S}_{\phi}(v \tau) & \text { if } v \tau<<D\end{cases}$ 

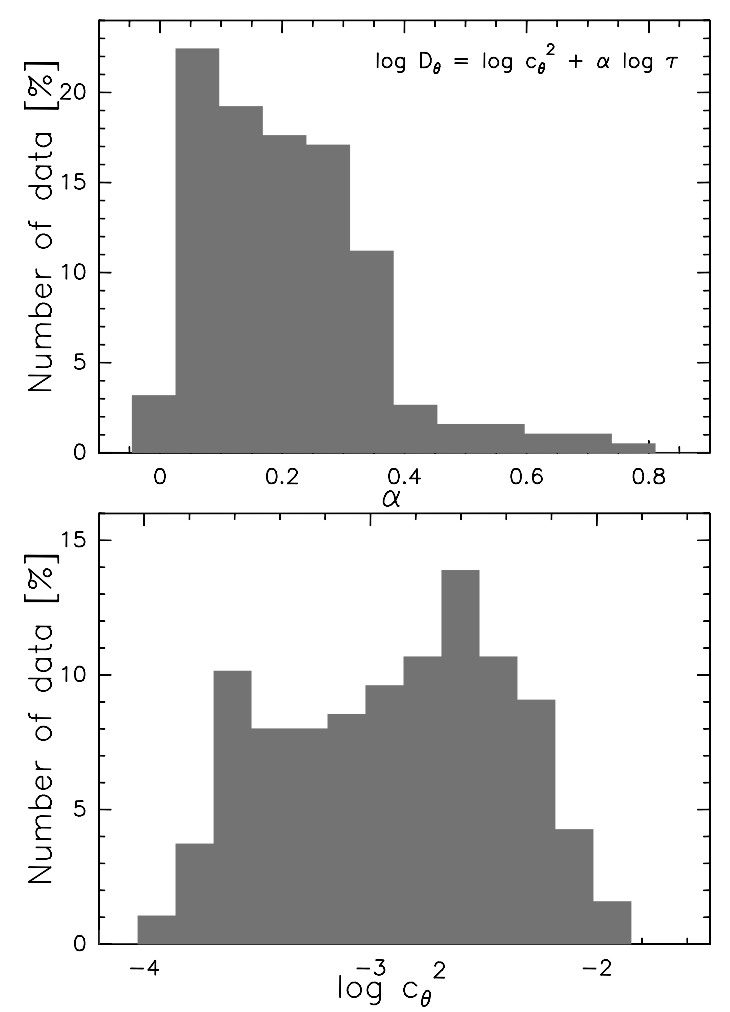

Fig. 6. Histograms showing the distribution of $\alpha$, the slope of the structure function (top), and of the intercept, $\log c_{\theta}{ }^{2}$ (bottom), for data sample I and II.

where $\mathcal{D}_{\theta}(\tau)$ is independent of $\tau$ for large values of $v \tau$, corresponding to saturation of the structure function, and $\mathcal{D}_{\theta}(\tau)$ can be fit with a power law for small values of $v \tau$. The break in the slope of $\mathcal{D}_{\theta}(\tau)$ occurs at lag times $\tau \sim$ $D / v \equiv \tau_{\mathrm{D}}$ when the telescope is pointed to the zenith. For Kolmogorov turbulence $\mathcal{S}_{\phi}(b)=c^{2} b^{5 / 3}$ and thus Eqs. (8) become:

$\mathcal{D}_{\theta}(\tau) \simeq \begin{cases}\frac{2 c^{2} D^{2 / 3}}{k} & \text { if } v \tau>>D \\ \frac{2 v^{5 / 3} c^{2}}{k D} \tau^{5 / 3} & \text { if } v \tau<<D\end{cases}$

and comparing with Eq. (1) we obtain:

$$
c_{\theta}^{2}=\frac{2 v^{5 / 3} c^{2}}{k D}
$$

where $c^{2}$ has units of $\mathrm{m}^{-5 / 3}$ and thus $c_{\theta}^{2}$ has units of $\mathrm{s}^{-5 / 3}$.

A comparison between Eq. (9) and Fig. 6 shows that the measured value of $\langle\alpha\rangle=0.2$ is much smaller than the Kolmogorov value $\alpha \simeq 1.67$. However, the distribution in Fig. 6 could be explained if most of the data were taken near or at saturation, i.e. $\tau \gtrsim \tau_{\mathrm{D}}$, or if Taylor's hypothesis is not entirely correct. If we use a typical value of $v_{\mathrm{w}} \simeq$ $5 \mathrm{~m} / \mathrm{s}$ for the wind speed parallel to the ground and a zenith angle of $z=45^{\circ}$, and if we also assume that the wind speed vector and the line of sight lie on the same plane, then:

$\tau_{\mathrm{D}}=\frac{D}{v_{\mathrm{w}}} \sec z \simeq 3.9 \mathrm{~s}$.

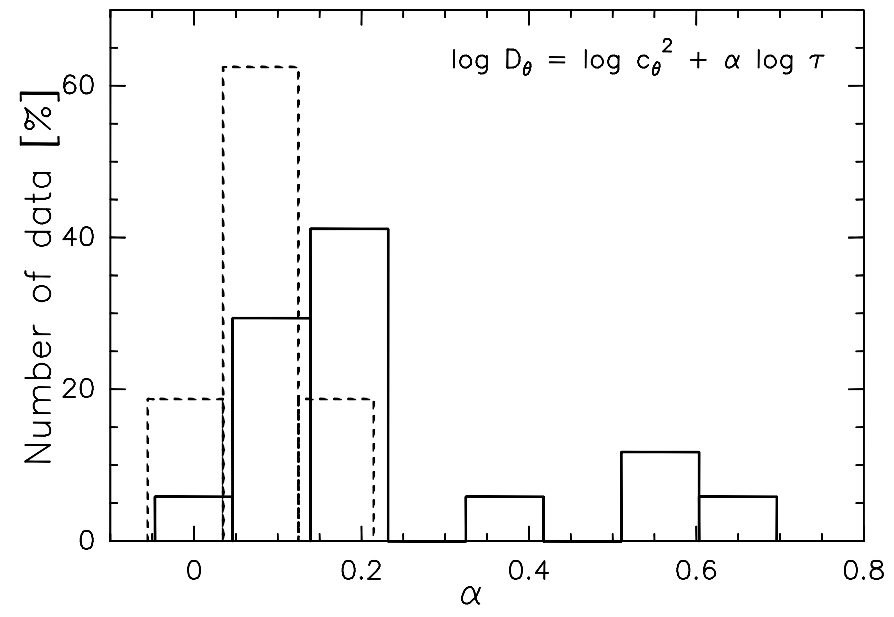

Fig. 7. Histogram showing the distribution of $\alpha$ at elevations $<30^{\circ}$ (solid line) and at elevations $>50^{\circ}$ (dashed line).

We thus see that using typical observing parameters $\tau_{\mathrm{D}} \gtrsim$ $\tau_{\mathrm{s}}$. Moreover, steeper slopes of the structure function should be observed more frequently at low wind speed and/or small elevation angles.

In Fig. 7 we plot the histograms of $\alpha$ for the data with $E L<30^{\circ}$ and for those with $E L>50^{\circ}$, and one can see that the distribution of the data taken at lower elevations is skewed towards higher $\alpha$ vales, as confirmed by their weighted averages of 0.23 and 0.08 for the two histograms, respectively. The data taken at higher elevations have no values of $\alpha \gtrsim 0.2$.

Although Fig. 7 suggests that saturation (i.e., a smaller slope of the structure function) is more likely to be observed at higher elevation angles, we could not find any clear correlation between $\tau_{\mathrm{D}}$ and $\sec z$, as suggested by Eq. (11). This may be due to: (i) wind speed vector not coplanar, on average, with the telescope line of sight, and (ii) distribution of elevation angles biased towards intermediate values (see Fig. 1).

\subsection{Power spectra and Allan variance}

The one-sided power spectrum can also be calculated from the $\Delta \theta(t)$ time series, and it is defined as:

$P_{\theta}(f)=2\left|\mathcal{F}_{\theta}(f)\right|^{2} \propto f^{\beta}$

where $\mathcal{F}_{\theta}(f)$ is the Fourier transform of $\Delta \theta(t)$ and $f$ is the temporal frequency. Most of the power spectra can also be fit using a power law, hence the proportionality sign in Eq. (12). This is shown in Fig. 8 where we have plotted the power spectra of the same AR time series from sample II whose structure functions were presented in Fig. 5. Despite the noise it is clear that the power spectrum for the day 13-APR-2000 cannot be fit with a single line and that a break in the slope of the spectrum occurs at a frequency of about $0.05 \mathrm{~Hz}$, consistent with the time lag corresponding to the beginning of saturation in the structure function shown in the top panel of Fig. 5 . 


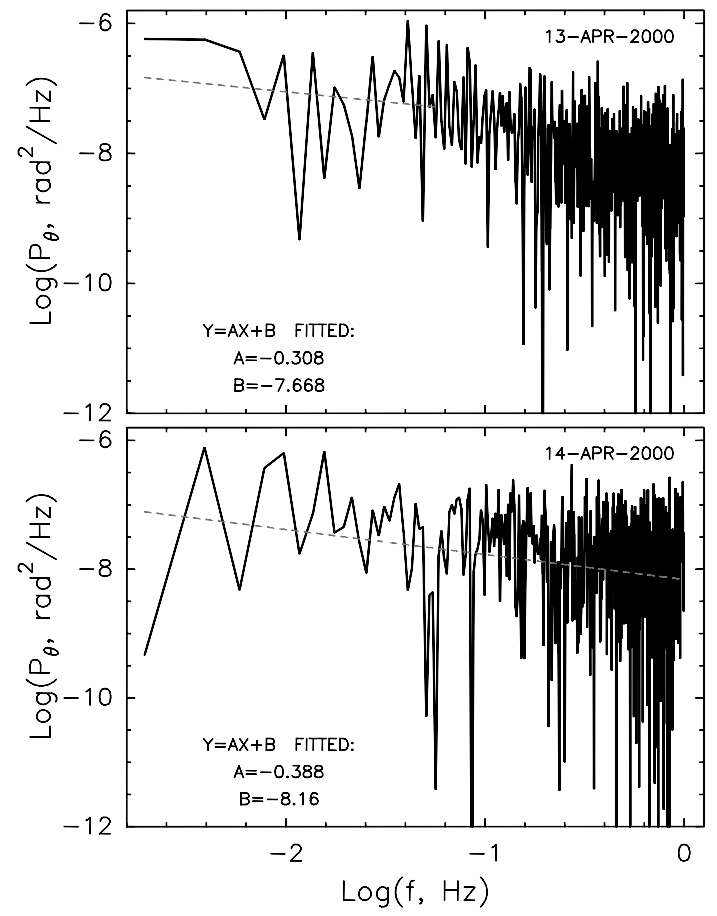

Fig. 8. Power spectra of the same time series whose structure functions are shown in Fig. 5. In the top-panel a break in the slope of the spectrum can be seen at a frequency of about $0.05 \mathrm{~Hz}$.

It can be shown that if the interferometer phase structure function $\mathcal{S}_{\phi}(b) \propto b^{\alpha}$ then the one-dimensional temporal phase power spectrum $P_{1}(f) \propto f^{-(1+\alpha)}$ (Armstrong $\&$ Sramek 1982). Because the slope of the phase structure function, $\mathcal{S}_{\phi}$, is the same as the slope of the AR structure function, $\mathcal{D}_{\theta}$, as shown by Eq. (9), one should expect that if $\mathcal{D}_{\theta}(\tau) \propto \tau^{\alpha}$ then $P_{\theta}(f) \propto f^{-(1+\alpha)}$ (see Sect. 3.5 for a discussion of this point). The weighted average of the slopes of the AR power spectra is -0.73 , and we can compare this value with the model of the angle of arrival spectrum obtained by Fante (1975). If we use Fante's Eq. (84) with $v=5 \mathrm{~m} / \mathrm{s}$ we find that in the frequency range $0.004-0.16 \mathrm{~Hz}$ the slope is $\simeq-0.8$, consistent with the AR average value. The intensity values of the power spectra (e.g., for the examples shown in Fig. 8) are also consistent with Fante's model assuming the turbulence outer scale is $L_{\circ} \simeq 500 \mathrm{~m}$ and $C_{\mathrm{n}}^{2} L \simeq 5 \times 10^{-8} \mathrm{~m}^{-1 / 3}$ (see Sect. 3.5 for a discussion on the parameter $C_{\mathrm{n}}^{2} L$ ).

The Allan variance is another useful method to describe the atmopsheric phase fluctuations (see, e.g., Armstrong \& Sramek 1982; Thompson et al. 1986; Olmi \& Downes 1992; Wright 1996). The Allan variance of the AR fluctuations removes linear drifts from the data and is defined as:

$$
\sigma_{\mathrm{y}}^{2}=\frac{1}{2(\pi \nu \tau)^{2}}\left\langle\left[\frac{\theta(t+2 \tau)+\theta(t)}{2}-\theta(t+\tau)\right]^{2}\right\rangle \propto \tau^{2 \gamma}
$$

where $\nu$ is the observing frequency. The Allan variance is related to the one-dimensional power spectrum and it

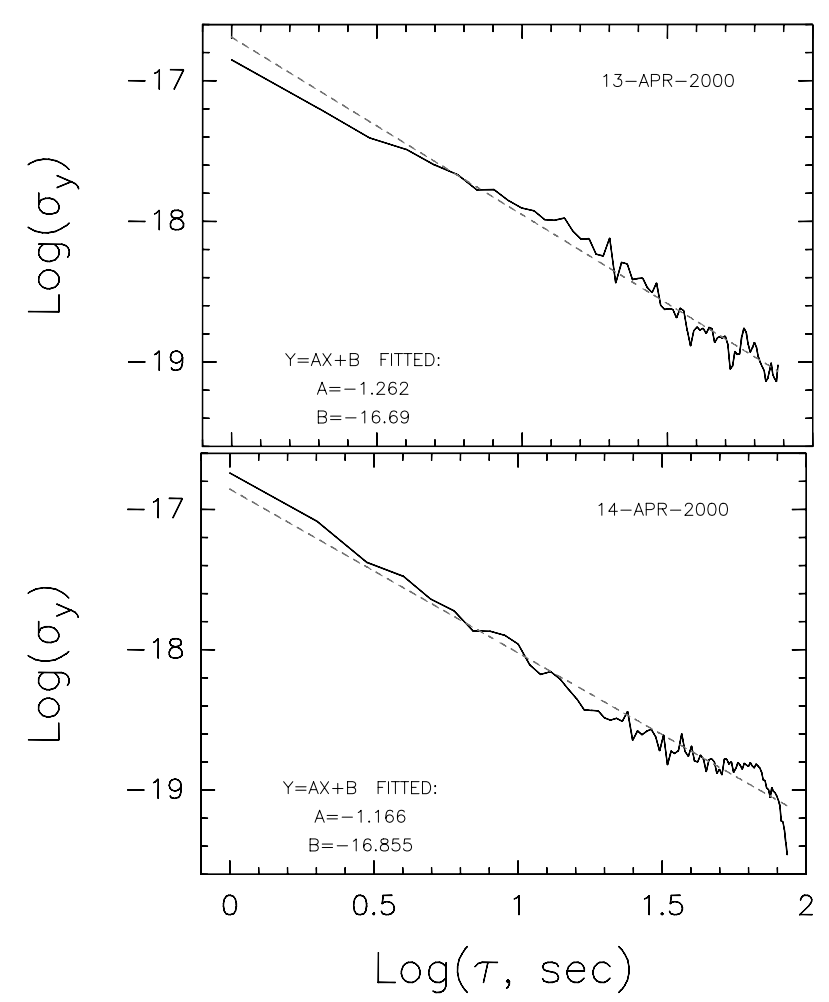

Fig. 9. Allan standard deviations of the same time series whose structure functions are shown in Fig. 5.

can be shown that if the structure function can be described with a power law of slope $\alpha$ then $\sigma_{\mathrm{y}} \propto \tau^{\gamma}$ where $\gamma=(\alpha-2) / 2$ (Armstrong \& Sramek 1982; Thompson et al. 1986) (see also Sect. 3.5). Two examples of Allan standard deviations are shown in Fig. 9, where we have plotted $\sigma_{\mathrm{y}}$ for the same AR time series whose structure functions and power spectra are shown in Figs. 5 and 8, respectively. We can see that the plot of $\sigma_{\mathrm{y}}$ for the data of 13-APR-2000 also shows a change in the slope, as it was already the case for the power spectrum shown in Fig. 8, and as a consequence the fit to the whole data is not as good as in the example of 14-APR-2000.

\subsection{Correlations}

We have carried out a statistical analysis of the data to identify possible correlations of either the power law indices or $\log c_{\theta}{ }^{2}$ with selected observing parameters such as $P W V$, time of day, season and elevation angle.

Because as we mentioned earlier in Sect. 3.1 the $P W V$ is one of the major environmental factors associated with the magnitude of the AR errors, we first present in Fig. 10 the correlation of $\log c_{\theta}{ }^{2}$ with $P W V$. From the fit to our data we find the empirical formula:

$\log c_{\theta}^{2}=0.13 P W V-3.57$

with $c_{\theta}^{2}$ expressed in SI units and $P W V$ in $\mathrm{mm}$. In Fig. 10 we can see that the winter data, located in the region of lower $P W V$, have also lower $\log c_{\theta}^{2}$ values whereas the summer data are characterized by both higher $P W V$ and 


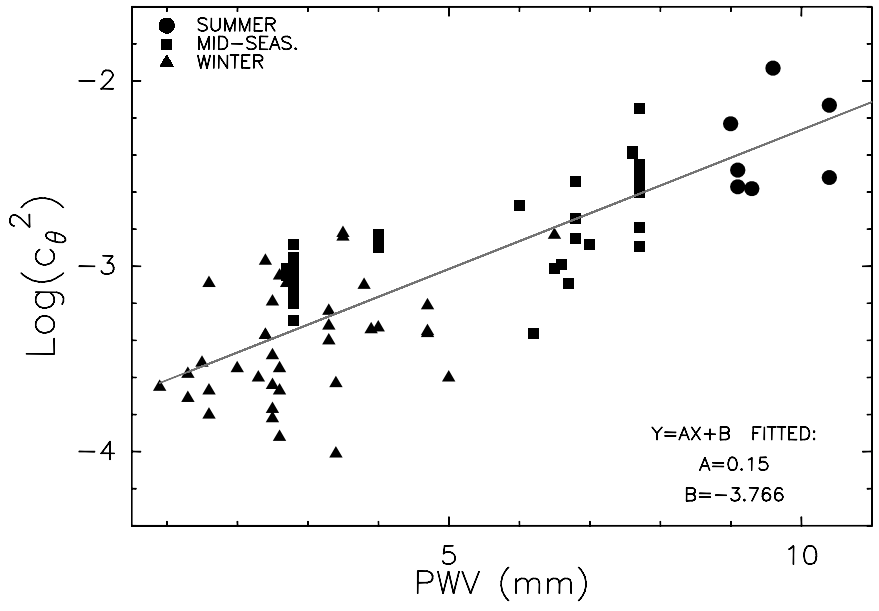

Fig. 10. Plot of $\log c_{\theta}^{2}$ vs. $P W V$.

$\log c_{\theta}{ }^{2}$ values. The mid-season data occupy the intermediate region of the plot.

We have previously seen in Eq. (10) that the structure coefficient of AR is proportional to the phase structure coefficient, which can be written for Kolmogorov turbulence $(\alpha=5 / 3)$ as (see, e.g., Roggemann \& Welsh 1996):

$c^{2}=2.91\left(\frac{2 \pi}{\lambda}\right)^{2} C_{\mathrm{n}}^{2} L$

where $C_{\mathrm{n}}^{2}$ is called the refractive index structure parameter, assumed constant up to a height $L$ and zero thereafter. It can be shown that at radio wavelengths the real part of $C_{\mathrm{n}}^{2}$, which determines the phase delay in an radio wave propagating through the atmosphere, is proportional to $\left\langle(\delta Q)^{2}\right\rangle /\langle Q\rangle^{2}$, i.e. the relative humidity fluctuations (Hill et al. 1980, 1988; Olmi 1994). Thus, the correlation shown in Fig. 10 might be explained if these fluctuations get stronger under conditions of higher humidity, which are typical of warmer weather when strong thermal gradients create considerable ground-level turbulence. As discussed by Coulman (1991) the morning and early afternoon heating disperses the nocturnal "inversions" because of vertical motion of convective elements, which may also explain the variation of $\log c_{\theta}^{2}$ with time shown in Fig. 13. Figure 11, which shows a clear correlation of $\log c_{\theta}^{2}$ with the groundlevel temperature, also suggests that increased AR fluctuations are in fact associated with increased convective activity near the ground. Figures 10 and 11 indicate that an increase in the humidity does not by itself lead to an increase in AR activity, as also discussed by Wright (1996) in regards of interferometer phase fluctuations, but that both are associated with larger $\left\langle(\delta Q)^{2}\right\rangle /\langle Q\rangle^{2}$ fluctuations as a result of an increased tropospheric turbulence.

In Fig. 12 we show $\log c_{\theta}{ }^{2}$ and $\alpha$ plotted as a function of $\log (\sec z)$. Because $\log c_{\theta}{ }^{2}$ shows a correlation with the local time (see Fig. 13) we selected only data in the 13:00 to 16:00 time interval in the top panel of Fig. 12 . However, because $\alpha$ does not seem to correlate with time (see discussion below) we used all data in the bottom panel of Fig. 12. Despite some big error bars it is clear from

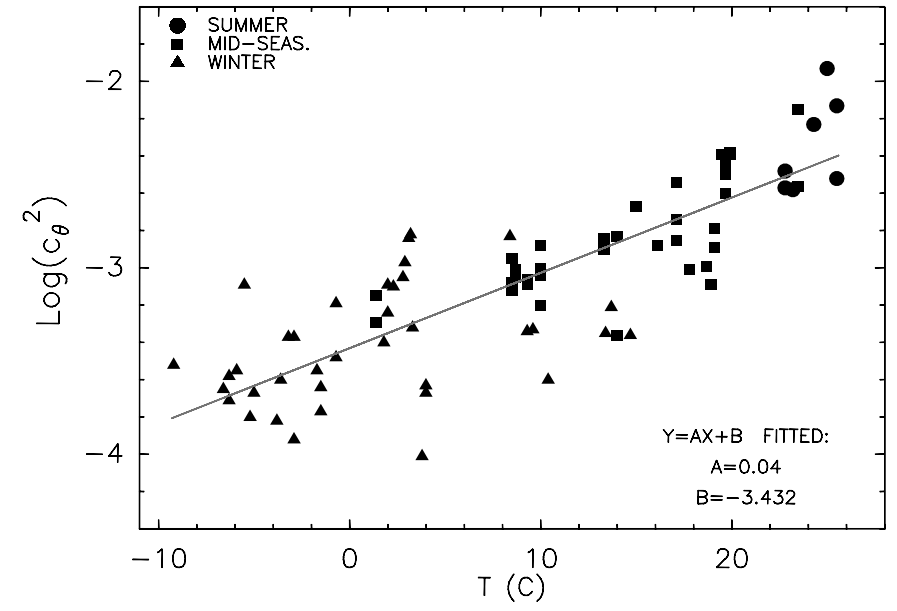

Fig. 11. Plot of $\log c_{\theta}^{2}$ vs. ground-level temperature.

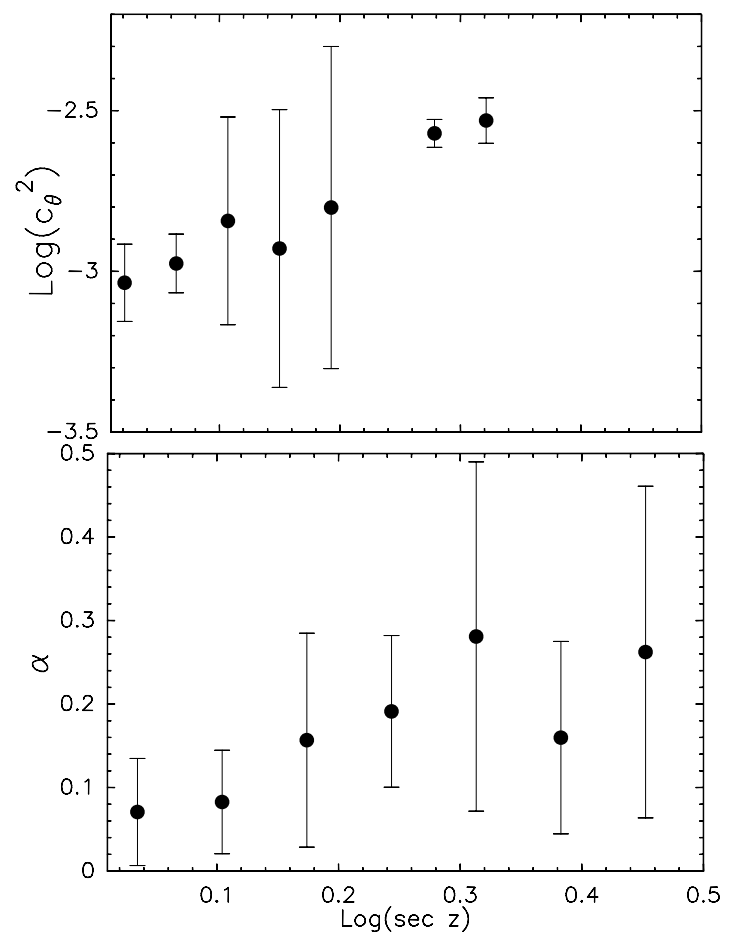

Fig. 12. Plots of $\log c_{\theta}^{2}$ (top) and $\alpha$ (bottom) vs. $\log (\sec z)$. Only data in the 13:00 to 16:00 time interval have been used in the top panel. The data have been binned to reduce the scatter.

Fig. 12 that the AR structure coefficient tends to increase at larger zenith angles. This trend was expected since for non-zenith angles Eq. (1) must be rewritten as:

$\mathcal{D}_{\theta}(\tau)=c_{\theta}^{2} \tau^{\alpha} \sec z$

as shown by Coulman (1991) and Olmi \& Downes (1992). Some decorrelation is expected to occur because the beamwidth changes with elevation angle, causing an error in retrieving the pointing error from the fluctuations in the source intensity, but because the beam $F W H M$ changes by $\pm 8 \%$ at most (see Sect. 2) this effect is negligible. The correlation of $\alpha$ with $\sec z$ is less clear, as shown in the bottom panel of Fig. 12. If subsequent observations will 


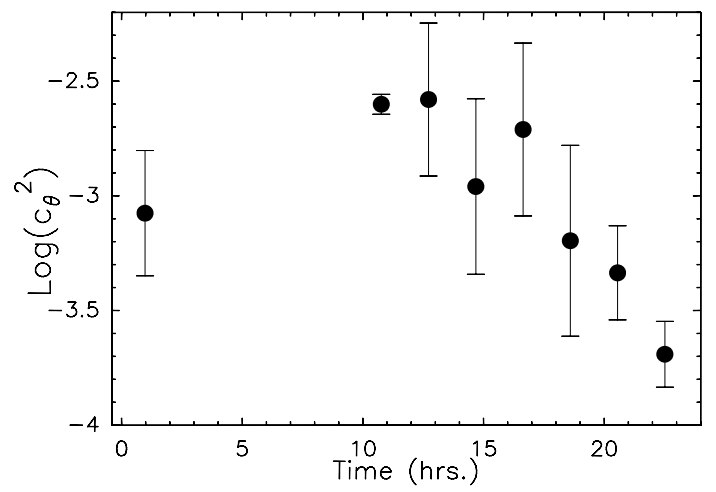

Fig. 13. Plot of $\log c_{\theta}{ }^{2}$ vs. local time. The selected data in the elevation range $30^{\circ}-60^{\circ}$ have been grouped into $2 \mathrm{hrs}$ bins to reduce the scatter. No selection based on the $P W V$ or ambient temperature values has been applied.

confirm that $\alpha$ tends to get bigger at smaller elevations, this would suggest that on the average the magnitude of the AR-induced pointing fluctuations, for a fixed time lag, becomes larger at smaller elevation angles.

We then wanted to find out whether a correlation exists between $\log c_{\theta}^{2}$ and the local time. We present our results in Fig. 13 where it can be clearly seen that the AR structure coefficient tends to decrease during the afternoon and evening hours. The night hours are less well sampled, and in particular we have no data between approximately 05:00 and 09:00, as shown in Fig. 1; however, the data suggest that a $\log c_{\theta}{ }^{2}$ peak may be reached sometime before 10:00. The day-night cycle of $\log c_{\theta}{ }^{2}$ follows a similar pattern of both $P W V$ and ambient temperature, and thus it seems to suggest that this diurnal variation is associated with increased turbulence near the ground during the day-time, as we suggested earlier in this section. A variability of the structure coefficient with time was also found by Olmi \& Downes (1992) in the case of the phase structure function measured with the IRAM interferometer. On the other hand, we find no clear correlation between $\alpha$ and the local time whereas such a correlation was also observed by Olmi \& Downes.

In Fig. 14 we plot the indices of the power laws describing the power spectrum and the Allan variance, defined in Eqs. (12) and (13), as a function of the power law index describing the structure function (sample II only). Clearly, $\beta$ and $\gamma$ correlate with $\alpha$, and the two best-fit lines are given by the equations $\beta=-1.43 \alpha-0.55$ and $\gamma=0.88 \alpha-1.29$, which are consistent with the predicted values of $\beta=-(1+\alpha)$ and $\gamma=0.5 \alpha-1$, respectively, as described in Sect. 3.4. This consistency between the measured best-fit values and the theoretical values of the various power law slopes, obtained applying the standard model of turbulence to the phase fluctuations of an interferometer, suggests that the same model can also be applied to the AR fluctuations measured with a singledish antenna. In fact, in Sect. 3.3.2 we showed how the structure functions of the phase and AR fluctuations can be related.

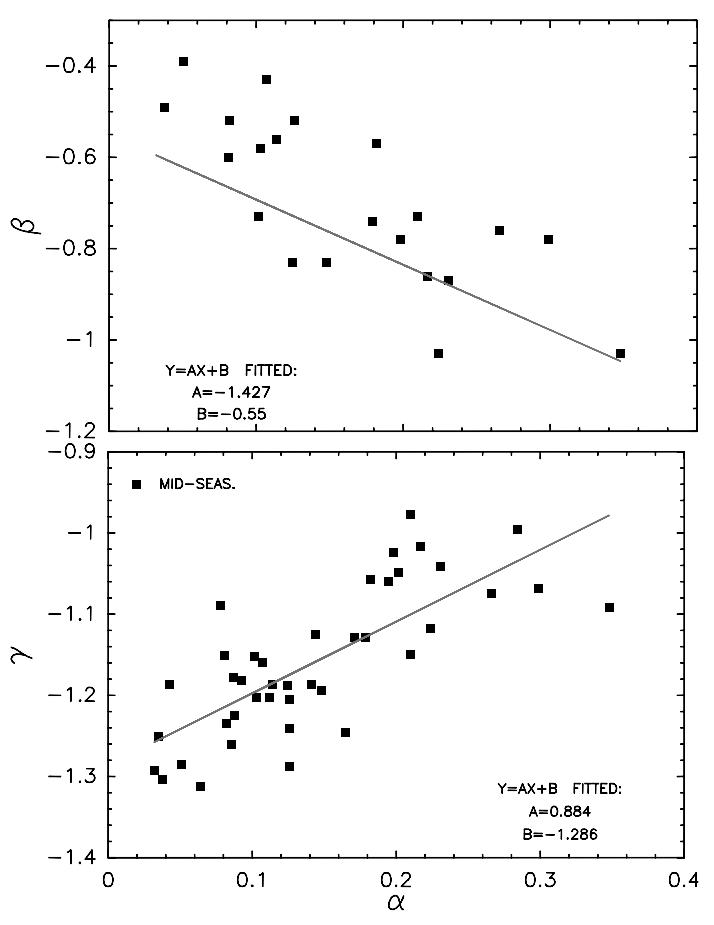

Fig. 14. Plots of $\beta$, the power law index of the power spectrum (top), and of $\gamma$, the power law index of the Allan variance (bottom), vs. $\alpha$, the power law index of the structure function.

\section{Conclusions}

We have carried out systematic measurements of ARinduced pointing errors with the radome-enclosed FCRAO $13.7 \mathrm{~m}$ telescope located in western Masschusetts on a flat terrain, during the period February 1999 to June 2000. The data are based on the time series of the fluctuations of the angular distance of the source from the beam center, as measured at the $-3 \mathrm{~dB}$ points. We have detected AR-induced pointing errors with the FCRAO 60" beam. The measured values range from $\simeq 2^{\prime \prime}$ (winter) to $\simeq 20^{\prime \prime}$ (summer). The probability density distributions of the AR pointing errors are narrower for low $P W V$ and wider for high $P W V$, and during typical dry weather $(P W V<4 \mathrm{~mm})$ the $F W H M$ of the distributions can be of several arcseconds. We have also measured the duration of the individual "AR events" and found that most of them last less than 3-4 s, with tails in the distribution stretching to $\simeq 10-20 \mathrm{~s}$. Such short-duration fluctuations will not be averaged out during a typical OTF scan and can thus affect the reliability of OTF mapping on ARlimited telescopes.

Several statistical functions have been used to analyse the data. We found that many structure functions can be fit with a single power law of type $\mathcal{D}_{\theta}(\tau)=$ $c_{\theta}^{2} \tau^{\alpha}$, where usually $\alpha<0.4$ and $\log c_{\theta}^{2} \sim-4$ to -2 . The slope of the AR structure functions is much lower than that of the phase structure functions measured with millimeter-wave interferometers. Power spectra and Allan variance plots can also be fit with single power laws, and we found that the three different power law slopes correlate and are consistent with the standard model of 
atmospheric turbulence. The magnitude of the AR fluctuations, represented by the structure coefficient, $c_{\theta}^{2}$, correlates well with $P W V$ and ground-level temperature, decreases with increasing elevation angle and also varies during the day. These characteristics indicate that stronger AR fluctuations are associated with increased convective activity near the ground, which is typical of warmer, and more humid, weather when strong thermal gradients create considerable ground-level turbulence. Correlations of $\alpha$ with the observing parameters are still unclear.

Extrapolation of these results to other telescope sites, such as the LMT/GTM site, is uncertain because of the different latitude, elevation and terrain characteristics. However, it seems reasonable to expect similar AR effects during conditions of similar $P W V$ and ambient temperature. If this is indeed the case then the expected magnitude of the AR-induced pointing errors can be comparable with the beam width of the LMT/GTM, under certain conditions, and all antenna measurements (OTF mapping, pointing, focusing, beam switching, etc.) would then be seriously affected. The LMT/GTM is currently studying the design of a radiometric wave front sensor to compensate AR effects.

Acknowledgements. This work was sponsored by the Advance Research Project Agency, Sensor Technology Office DARPA Order No. C134 Program Code No. 63226E issued by DARPA/CMO under contract No. MDA972-95-C-0004, and by the NSF grant AST-9725951. The author thanks M. Brewer and M. Heyer of FCRAO for help with the observing technique and reduction of some of the data used in this work.

\section{References}

Altenhoff, W. J., Baars, J. W. M., Downes, D., \& Wink, J. E. 1987, A\&A, 184, 381

Armstrong, J. W., \& Sramek, R. A. 1982, Radio Sci., 17, 1579

Bieging, J. H., Morgan, J., Welch, W. J., Vogel, S. N., \& Wright, M. C. H. 1984, Radio Sci., 19, 1505

Bremer, M. 1995, IRAM internal report, No. 238

Butler, B. 1997, internal MMA memo, No. 188

Church, S., \& Hills, R. 1990, in URSI/IAU Symp. Proc.,
Radioastronomical Seeing, ed. J. E. Baldwin, \& W. Shouguan

Close, L. M., \& Mc Carthy, D. W. Jr. 1994, PASP, 106, 77

Coulman, C. E. 1991, A\&A, 251, 743

Downes, D., \& Altenhoff, W. J. 1990, in URSI/IAU Symp. Proc., Radioastronomical Seeing, ed. J. E. Baldwin, \& W. Shouguan

Fante, R. L. 1975, Proc. of the IEEE, 63, 1669

Hill, R. J., Clifford, S. F., \& Lawrence, R. S. 1980, J. Opt. Soc. Am., 70, 1192

Hill, R. J., Bohlander, R. A., Clifford, S. F., et al. 1988, IEEE Trans. Geosc. Rem. Sens., 26, 330

Holdaway, M. A. 1997, internal MMA memo, No. 186

Holdaway, M. A., \& Woody, D. 1998, internal MMA memo, No. 223

Kaercher, H. J., \& Baars, J. W. M. 2000, Proc. of the SPIE, Astronomical telescopes and instrumentation 2000, ed. H. R. Butcher, vol. 4015, 155

Ladd, N., \& Heyer, M., 28 October 1996, FCRAO Technical Memorandum

Lawrence, R. S., \& Strohbehn, J. W. 1970, Proc. of the IEEE, 58,1523

Lay, O. 1997, A\&AS, 122, 535

Marvel, K. B., \& Woody, D. P. 1998, Proceedings of the SPIE, Astronomical telescopes and instrumentation, ed. T. G. Phillips, vol. 3357, 442

Olmi, L. 1994, Proc. of the International Meeting, Microwave Radiometry and Remote Sensing of the Environment, ed. D. Solimini (VSP, International Science Publishers, The Netherlands), 79

Olmi, L. 1998, Proc. of the SPIE, Astronomical telescopes and instrumentation, ed. T. G. Phillips, vol. 3357, 186

Olmi, L. 2000a, Radio Sci., 35, 275

Olmi, L. 2000b, Proc. of the SPIE, Astronomical telescopes and instrumentation 2000, ed. H. R. Butcher, vol. 4015, 390

Olmi, L. 2001, Proc. of the IAU Site 2000 Workshop, held in Marrakech, Morocco, 13-17 November 2000, in press

Olmi, L., \& Downes, D. 1992, A\&A, 262, 634

Roggemann, M. C., Welsh, B. 1996, in Imaging through turbulence (CRC press, Boca Raton USA)

Thompson, A. R., Moran, J. M., \& Swenson, G. W. 1986, in interferometry and synthesis in radio astronomy (J. Wiley \& sons, New York, USA)

Wright, M. C. H. 1996, PASP, 108, 520 